\title{
DESIGN AND EVALUATION OF A NEW AUDITORY DISPLAY FOR THE PULSE OXIMETER
}

\author{
Estrella Paterson \\ Penelope Sanderson \\ Neil Paterson \\ The University of Queensland \\ Sir Fred Schonell Drive \\ St Lucia, Queensland 4051, Australia \\ estrella.paterson@uqconnect.edu.au
}

\begin{abstract}
During surgery the pulse oximeter device provides information about a patient's oxygen saturation $\left(\mathrm{SpO}_{2}\right)$ and heart rate via visual and auditory displays. An audible tone is emitted after every detected pulse (indicating heart rate), and the pitch of the tone is mapped to $\mathrm{SpO}_{2}$. However, clinicians cannot reliably judge $\mathrm{SpO}_{2}$ using only the current auditory display. In a series of three studies, we compared auditory displays based on current pulse oximeters with displays designed to provide more information about $\mathrm{SpO}_{2}$ levels using additional acoustic properties. Results from the first two laboratory studies show that the new auditory displays support better identification of specified ranges of $\mathrm{SpO}_{2}$, and better detection of when saturation transitions a critically relevant threshold. The analysis of a third study in a highfidelity simulator is currently under way. An auditory display that provides more information about $\mathrm{SpO}_{2}$ levels and when $\mathrm{SpO}_{2}$ changes from one range to another may be useful for clinicians when they are engaged in other visually demanding tasks but have to detect and treat patient deterioration, often in time-pressured and stressful situations.
\end{abstract}

\section{INTRODUCTION}

Over the past three decades, the pulse oximeter (PO) has become standard equipment in a number of hospital settings including the operating room (OR), recovery room, intensive care unit and patient transport[1]. It provides a visual display (numerical and waveform) and an auditory display (variable pitch plus alarms) of the patient's oxygen saturation $\left(\mathrm{SpO}_{2}\right)$, heart rate, and heart rhythm.

The auditory display is especially important when clinicians are engaged in other visually demanding tasks, when the visual display is obscured, or when visual overload occurs [2-4]. During an operation, anaesthetists are usually not looking at the visual display; they look at the display only around $5-30 \%$ of intraoperative time $[5,6]$. Thus, clinicians depend on the auditory display to provide patient information. However, clinicians cannot reliably identify $\mathrm{SpO}_{2}$ levels accurately using the current auditory display alone [7-9]. The current auditory display is based on tones of variable pitch, supplemented with alarms set at a clinically relevant threshold. As $\mathrm{SpO}_{2}$ decreases from a maximum of $100 \%$, the pitch of the tones decreases. Although people find it easy to recognise pitch changes, very few people have absolute pitch [10] making it difficult to identify $\mathrm{SpO}_{2}$ values using pitch alone.

The problem of identifying $\mathrm{SpO}_{2}$ from the pulse oximeter tone is exacerbated by a number of factors. First,

(c) (1) (8) This work is licensed under Creative Commons Attribution Non Commercial 4.0 International License. The full terms of the License are available at http://creativecommons.org/licenses/by-nc/4.0

\author{
Robert Loeb
}

\author{
Department of Anesthesiology \\ University of Florida College of Medicine \\ Gainsville, FL 32610, USA \\ RLoeb@anest.ufl.edu
}

clinicians have many tasks to perform while monitoring patients [11] and therefore have to divide attention between these tasks and patient monitoring. Second, the OR can be a noisy environment. Research shows that as noise levels increase, anaesthetists' ability to distinguish between $\mathrm{SpO}_{2}$ levels diminishes [9]. Third, during surgical procedures, anaesthetists are frequently interrupted and distracted [12].

In a series of three studies, devised as incremental design experiments, we evaluated a new auditory display for the pulse oximeter. Our aim was to test whether the new auditory display better supports judgements about $\mathrm{SpO}_{2}$ range, and when the $\mathrm{SpO}_{2}$ changes from one range to another, than does a standard display. Visual display of $\mathrm{SpO}_{2}$ was not provided in any of the three experiments.

In the first laboratory study we compared the ability of non-clinician participants to identify $\mathrm{SpO}_{2}$ levels using five different auditory pulse oximetry displays, including a standard display similar to those in current use, while they performed a visual distractor task[13]. In the second laboratory study we compared the ability of clinician and non-clinician participants to identify $\mathrm{SpO}_{2}$ levels using the standard display and the best enhanced display from Study 1, while they also performed visual and auditory distractor tasks. Finally, in our current study, we are comparing anaesthetists' ability to distinguish $\mathrm{SpO}_{2}$ levels in a highfidelity simulator using the displays from Study 2.

\section{STUDY 1}

In former studies, we found that listeners can distinguish $\mathrm{SpO}_{2}$ levels more accurately when the pulse oximeter's variable pitch tone is enhanced with tremolo and acoustic brightness than when variable pitch alone is used [14, 15]. This may be because listeners can more easily use auditory displays with multiple heterogeneous features indicating state changes than displays with only one such feature [16]. A limitation was that participants' only task was to judge $\mathrm{SpO}_{2}$ levels, whereas in the OR anaesthetists have many tasks to perform while monitoring patients' states. Furthermore, the experiments were conducted in a quiet room. Noise levels in the OR average 51-75 dB [17] and can reach levels of $120 \mathrm{~dB}[18]$.

In the current Study 1, using a between-subjects design, we measured 100 non-clinician participants' accuracy and latency at detecting transitions into and out of an $\mathrm{SpO}_{2}$ target range, identifying $\mathrm{SpO}_{2}$ range (target, low, critical), and identifying the absolute $\mathrm{SpO}_{2}$ value, using five different auditory displays[13]. We addressed limitations of the above studies by including a secondary distractor task (arithmetic verification) plus background noise. 


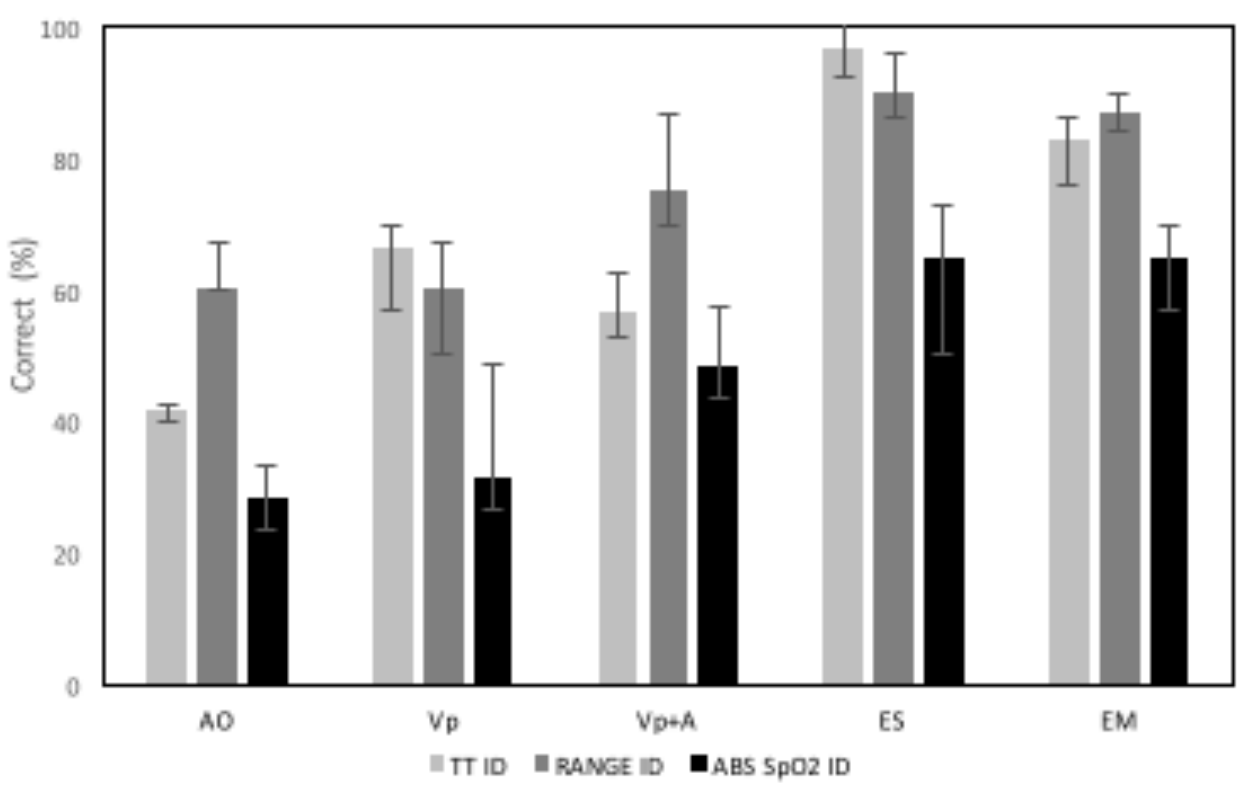

Figure 1. Participants' accuracies of Target Transition identification, Range identification, and Absolute $\mathrm{SpO}_{2}$ Value identification, for Alarm only (AO), Varying pitch(Vp), Varying pitch plus alam (Vp+A), Enhanced single (ES) and Enhanced Multiple (EM) conditions. (Mean $\pm \mathrm{CI}$ )

Background noise contained dialogue, OR noises and pop music with vocals.

We tested $\mathrm{SpO}_{2}$ values from $100 \%-80 \%$ and divided them into three ranges: target $\left(100 \%-97 \% \mathrm{SpO}_{2}\right)$, low $(96 \%-90 \%$ $\left.\mathrm{SpO}_{2}\right)$ and critical $(89 \%-80 \% \mathrm{SpO} 2)$. The five auditory displays are described in detail below and in this sound file: https://www.dropbox.com/sh/r8xfqiyyeto2r1w/AACgLdHfeMAb6JvJu-9hU69a?dl=0

The control condition, Variable pitch plus alarm $(V p+A)$, was based on the auditory display of current pulse oximeters and comprised variable pitch pulse tones with an audible alarm set at $89 \% \mathrm{SpO}_{2}$ [19]. Tones were sine wave functions ranging logarithmically from $150 \mathrm{~Hz}$ at $80 \% \mathrm{SpO}_{2}$ to $950 \mathrm{~Hz}$ at $100 \%$ $\mathrm{SpO}_{2}$; Each tone lasted for $150 \mathrm{~ms}$ with a $10 \mathrm{~ms}$ fade-in and $10 \mathrm{~ms}$ fade-out to eliminate acoustic artefact.

In the Alarm only (AO) condition there were no pulse tones; only an alarm [IEC-Medium-General alarm (IEC-606011-8)] that sounded when $\mathrm{SpO}_{2}$ entered the critical range from the low range and every $15 \mathrm{~s}$ thereafter that $\mathrm{SpO}_{2}$ remained in the critical range. This condition represents typical use of the auditory display used in the intensive care unit, with the variable pitch deactivated to reduce noise levels.

The Variable pitch (Vp) condition was the same as the control condition but without an alarm. This condition corresponds to use of pulse oximetry displays when the alarm is silenced or alarm limits are set very wide.

The first experimental condition for single patient monitoring, Enhanced single (ES), comprised the same variable pitch mapping as the control condition but with tremolo added to tones in the low and critical ranges $(96 \%-$ $\left.80 \% \mathrm{SpO}_{2}\right)$ and brightness added in the critical range $(89 \%$ $80 \% \mathrm{SpO}_{2}$ ). Tremolo was produced by modulating the peak amplitude of the tone: four cycles of tremolo with $90 \%$ wet. Brightness was produced by adding odd harmonics of the tone's fundamental frequency (third, fifth and seventh harmonic) to produce a sharper sound.
The second experimental condition, Enhanced multiple (EM) was the same as Enhanced single except that variable pitch tones were excluded when $\mathrm{SpO}_{2}$ was in the target range. Instead pulse tones were replaced by a chirp (an "all well" sound) that sounded every $5 \mathrm{~s}$ that $\mathrm{SpO}_{2}$ remained in the target range. The chirp had a duration of $100 \mathrm{~ms}$, started at $1000 \mathrm{~Hz}$ that decreased linearly to $500 \mathrm{~Hz}$ at the $50 \mathrm{~ms}$ midpoint and increased to $1000 \mathrm{~Hz}$ at the end of the tone. Volume increased from 0 to 0.3 (on a scale of $0-1$ ) at the midpoint and decreased to 0 at $100 \mathrm{~ms}$. This display represents a prototype that we have developed for monitoring multiple patients. When all patients' $\mathrm{SpO}_{2}$ remains in target range, only a series of chirps is heard. If $\mathrm{SpO}_{2}$ for one patient moves from the target range, the "all well" sound changes to the enhanced variable pitch tone.

Participants were trained to identify $\mathrm{SpO}_{2}$ range and absolute $\mathrm{SpO}_{2}$, values and to detect when $\mathrm{SpO}_{2}$ moved into or out of the target range (target transitions). They then completed two blocks of fifteen 60-second trials each.

Results are shown in Figure 1. Participants using either of the two experimental auditory displays enhanced with additional acoustic properties (ES and EM) were more accurate and faster at detecting target transitions, and more accurate at identifying $\mathrm{SpO}_{2}$ range and absolute $\mathrm{SpO}_{2}$ values, than participants using the Variable pitch plus alarm condition $(\mathrm{Vp}+\mathrm{A})$. Participants in the Alarm only condition were less accurate and slower at detecting target transitions, and less accurate at identifying $\mathrm{SpO}_{2}$ ranges and absolute $\mathrm{SpO}_{2}$ values that those in the $\mathrm{Vp}+\mathrm{A}$ condition. There was no difference for participants in the Variable pitch $(\mathrm{Vp})$ and $\mathrm{Vp}+\mathrm{A}$ condition for target transition detection accuracy or latency but participants in the $\mathrm{Vp}+\mathrm{A}$ condition were more accurate than those in the $\mathrm{Vp}$ condition for $\mathrm{SpO}_{2}$ range and absolute $\mathrm{SpO}_{2}$ identification accuracy.

This study provides evidence that auditory displays comprising variable pitch with additional acoustic properties of tremolo and brightness are more effective for identifying 
$\mathrm{SpO}_{2}$ levels than an auditory display similar to that of current pulse oximeters.

\section{STUDY 2}

Study 1 established the superiority of displays enhanced with additional acoustic properties over a standard display for $\mathrm{SpO}_{2}$ parameter identification. Participants performed only one distractor task that was presented visually, and participants were from a non-clinical population. However, many anaesthetic tasks involve verbal communication, some essential for effective team performance and some irrelevant to case management. Verbal processing may interfere with perception of the pulse oximeter's auditory signal. Furthermore, clinicians' greater familiarity with standard pulse oximetry auditory displays might mean they perceive the signal differently from non-clinicians. Thus, in Study 2, we added a distractor task in the same perceptual modality as the monitoring task, and tested non-clinician and clinician participants.

In a laboratory study using a counterbalanced, within and between-subjects, crossover design, non-clinician participants $(n=28)$ and specialist/trainee anaesthetists $(n=25)$ from a tertiary hospital identified $\mathrm{SpO}_{2}$ levels using the Variable pitch plus alarm (standard) and the Enhanced single (enhanced) displays from Study 1. Participants performed two distractor tasks simultaneously: arithmetic verification task from Study 1 and a new keyword detection task. Each participant performed the experiment over two blocks of 15 trials each: one using the standard display and the other using the enhanced display. Each trial lasted $60 \mathrm{~s}$ with heart rate set at $72 \mathrm{bpm}$. Participants received training before each block. Participants identified $\mathrm{SpO}_{2}$ target transitions during a trial, and $\mathrm{SpO}_{2}$ range and absolute $\mathrm{SpO}_{2}$ value at the end of each trial. Ranges were the same as in Study 1: target, low and critical.

For the keyword detection task, we designed 30 linguistic scenarios, one per trial. In each trial there were seven spoken phrases comprising 0-4 keyword phrases. Participants identified keywords: BLOOD, PATIENT or TABLE. Background noise contained OR noises, and music with vocals played throughout the experiment.

Participants were more accurate and faster at detecting $\mathrm{SpO}_{2}$ target transitions with the enhanced display $(87 \%, 2.4 \mathrm{~s})$ than with the standard display $(57 \%, 8.7 \mathrm{~s}), \mathrm{p}<.001$ for each measure. Participants were more accurate at identifying $\mathrm{SpO}_{2}$ range and absolute $\mathrm{SpO}_{2}$ value with the enhanced display $(86 \%, 66 \%)$ than with the standard display $(76 \%, 46 \%)$, $\mathrm{p}<.001$ for each measure. Participants reported that they found the monitoring task easier and were more confident of their judgements with the enhanced display than with the standard display. We found no differences between clinicians and nonclinicians for performance accuracies or speeds, or for subjective judgements.

This study provides additional evidence that an auditory display enhanced with tremolo and brightness is more effective for identifying $\mathrm{SpO}_{2}$ levels than a standard display using only pitch and alarms, even when participants are engaged in an auditory distractor task as well as a visual computational task. There was no difference in performance between clinicians and non-clinicians, which may not be surprising given that the experiment tested only perception and classification performance [20]

\section{STUDY 3}

Anaesthetists have many tasks to perform while they are continuously monitoring patients' states, and they are subject to numerous distractions and interruptions [11, 21]. Highfidelity simulators are powerful environments for investigating equipment usability in safety critical systems. They let investigators test devices in more challenging and authentic clinical settings, such as the OR. [22]. We designed a study to test whether the enhanced display would help anaesthetists monitor $\mathrm{SpO}_{2}$ levels more accurately compared with the standard display. We used the simulator suite at a large paediatric hospital, and set it up as an OR. Participants were consultant anaesthetists $(\mathrm{N}=20)$ who identified $\mathrm{SpO}_{2}$ levels using standard and enhanced displays from Study 2. In addition, participants identified changes in heart rate, blood pressure and $\mathrm{CO}_{2}$. Each participant performed two different experimental scenarios, one for each display and each lasting 20 minutes. Scenarios were counterbalanced across both displays, were deterministic, and were controlled from the simulator control room.

Participants were trained to use the auditory display before each scenario. They performed a cognitivelydemanding distractor task during each scenario: categorisation of patient details. Participants were also interrupted during scenarios, both directly and via telephone. All scenarios were video recorded. The video recordings will be coded for verbal responses relating to detection of $\mathrm{SpO}_{2}$ range transition (target to low and low to critical in both directions) and identification of $\mathrm{SpO}_{2}$ range. We are currently still analyzing the results of Study 3 , but early results are promising.

\section{GENERAL DISCUSSON AND CONCLUSION}

The purpose of this program of research was to evaluate a new auditory display for the pulse oximeter. In a series of three studies we tested listeners' ability to identify $\mathrm{SpO}_{2}$ levels using different auditory displays. In Study 1 we established that non-clinician participants detected $\mathrm{SpO}_{2}$ target transitions and identified $\mathrm{SpO}_{2}$ ranges and absolute $\mathrm{SpO}_{2}$ values more accurately using an auditory display enhanced with tremolo and brightness compared with a pitch plus alarm display. Participants performed these tasks while doing a visual distractor task and in the presence of simulated background OR noises. In Study 2 we found superiority of the enhanced auditory display held, even when participants performed the visual task, plus a keyword detection task presented in the same modality as the monitoring task. There was no difference between performance of clinicians and non-clinicians, indicating that the new display has potential for use by novices. In Study 3 we have tested whether the effect holds in the more realistic environment of a simulator.

The experimental display enhanced with tremolo and brightness for non-normal ranges provides more information about $\mathrm{SpO}_{2}$ levels than does the standard display of variable pitch plus alarm. In the first two experiments, when $\mathrm{SpO}_{2}$ transitioned the target-low threshold, participants were able to detect transitions using the enhanced display far more accurately and faster than when using the standard display. Such a display may enable clinicians to monitor patients preattentively and continuously, allowing attention to be directed to other visually demanding tasks. [23] The additional sound properties may attract auditory attention to pre-set thresholds, thus indicating a change in saturation levels so remedial action 
can be taken before a critical threshold is breached and an alarm sounds. This may help reduce the number of audible alarms and decrease annoyance from noise. The display may also help clinicians monitor whether treatment has been effective and detect exactly when $\mathrm{SpO}_{2}$ levels return to normal once more. These results may have implications for clinical practice. If clinicians can detect changes in $\mathrm{SpO}_{2}$ more accurately and faster they may be able to make decisions about treatment more effectively.

Our research shows that a PO auditory display enhanced with features such as tremolo and brightness to distinguish clinically important $\mathrm{SpO}_{2}$ ranges allows for more accurate judgment of $\mathrm{SpO}_{2}$ levels compared with displays similar to those of current pulse oximeters. If results from Study 3 show that $\mathrm{SpO}_{2}$ levels can still be distinguished much more effectively with the enhanced display than with the standard display in an environment similar to the OR, further clinical trials could be conducted. Importantly, commercial manufacturers and users would need to be consulted in evaluation of a new PO auditory display for it to be taken up successfully.

\section{ACKNOWLEDGMENTS}

We thank Dr Birgit Brecknell and Ismail Mohammed for software development, Dr Peter Moran, Princess Alexandra Hospital, Brisbane, and actors Isaac Salisbury, Jelena Zestic, Garry Mann, Felicity Burgmann, Tom Davidson, T-lok Tang and Lachlan Peterson (The University of Queensland, Brisbane)

\section{REFERENCES}

[1] A. Shah, and K. H. Shelley, "Is pulse oximetry an essential tool or just another distraction? The role of the pulse oximeter in modern anesthesia care," Journal of Clinical Monitoring and Computing, vol. 27, no. 3, pp. 235-242, 2013

[2] J. M. Ansermino, "Intelligent patient monitoring and clinical decision making," Monitoring Technologies in Acute Care Environments, M. C. J. M Ehrenfeld, ed., pp. 401-407, New York: Springer, 2014

[3] R. M. Craven, and A. K. McIndoe, "Continuous auditory monitoring--how much information do we register?," British journal of anaesthesia, vol. 83, no. 5, pp. 747, 1999.

[4] P. Sanderson, D. Liu, and S. A. Jenkins, "Auditory displays in anesthesiology," Current Opinion in Anesthesiology, vol. 22, no. 6, pp. 788-795, Dec, 2009.

[5] S. Ford, E. Birmingham, A. King, J. Lim, and J. M. Ansermino, "At-a-Glance Monitoring: Covert Observations of Anesthesiologists in the Operating Room," Anesthesia \& Analgesia, vol. 111, no. 3, pp. 653-658, 2010.

[6] C. M. Schulz, E. Schneider, L. Fritz, J. Vockeroth, A. Hapfelmeier, T. Brandt, E. F. Kochs, and G. Schneider, "Visual attention of anaesthetists during simulated critical incidents," British Journal of Anaesthesia vol. 106, no. 6, pp. 807-813, 2011.

[7] R. W. Morris, and P. J. Mohacsi, "How well can anaesthetists discriminate pulse oximeter tones?," Anaesthesia and Intensive Care, vol. 33, no. 4, pp. 497$500,2005$.

[8] G. T. Schulte, and F. E. Block, "Can people hear the pitch change on a variable-pitch pulse oximeter?,"
Journal of Clinical Monitoring, vol. 8, no. 3, pp. 198-200, 1992.

[9] R. A. Stevenson, J. J. Schlesinger, and M. T. Wallace, "Effects of divided attention and operating room noise on perception of pulse oximeter pitch changes: A laboratory study," Anesthesiology, vol. 118, no. 2, pp. 376-381, 2013.

[10] D. J. Levitin, and S. E. Rogers, "Absolute pitch: Perception, coding, and controversies," Trends in Cognitive Sciences, vol. 9, no. 1, pp. 26-33, 2005.

[11] D. Phipps, G. H. Meakin, P. C. Beatty, C. Nsoedo, and D. Parker, "Human factors in anaesthetic practice: Insights from a task analysis," British journal of anaesthesia, vol. 100, no. 3, pp. 333-343, 2008.

[12] M. van Pelt, and M. B. Weinger, "Distractions in the Anesthesia Work Environment: Impact on Patient Safety? Report of a Meeting Sponsored by the Anesthesia Patient Safety Foundation," Anesthesia and analgesia, vol. 85, no. 6, 2017.

[13] E. Paterson, P. M. Sanderson, N. A. B. Paterson, and R. Loeb, "The effectiveness of enhanced pulse oximetry sonifications for conveying oxygen saturation ranges: A laboratory comparison of five auditory displays," British Journal of Anaesthesia, vol. 119, no. 6, pp. 1224-30, 2017.

[14] K. Hinckfuss, P. Sanderson, R. G. Loeb, H. Liley, and D. Liu, "Novel pulse oximetry sonifications for neonatal oxygen saturation monitoring A laboratory study," Human Factors, vol. 58, pp. 344-359, 2016.

[15] E. Paterson, P. Sanderson, N. A. B. Paterson, D. Liu, and R. G. Loeb, "The effectiveness of pulse oximetry sonification enhanced with tremolo and brightness for distinguishing clinically important oxygen saturation ranges: A laboratory study," Anaesthesia, vol. 71, no. 5, pp. 565-572, 2016a.

[16] J. Edworthy, E. Hellier, K. Titchener, A. Naweed, and R. Roels, "Heterogeneity in auditory alarm sets makes them easier to learn," International Journal of Industrial Ergonomics, vol. 41, no. 2, pp. 136-146, 2011.

[17] D. Hasfeldt, E. Laerkner, and R. Birkelund, "Noise in the operating room-What do we know? A review of the literature," Journal of PeriAnesthesia Nursing, vol. 25, no. 6, pp. 380-386, 12//, 2010.

[18] J. M. Kracht, I. J. Busch-Vishniac, and J. E. West, "Noise in the operating rooms of Johns Hopkins Hospital," The Journal of the Acoustical Society of America, vol. 121, no. 5 Pt1, pp. 2673-2680, 2007.

[19] R. G. Loeb, B. Brecknell, and P. Sanderson, "The sounds of desaturation: A survey of commercial pulse oximeter sonifications," Anesthesia \& Analgesia, vol. 122, no. 5, pp. 1395-1403, 2016.

[20] J. Rasmussen, A. M. Pejtersen, and L. P. Goodstein, "Cognitive systems engineering," 1994.

[21] A. N. Healey, N. Sevdalis, and C. A. Vincent, "Measuring intra-operative interference from distraction and interruption observedin the operating theatre," Ergonomics, vol. 49, no. 5-6, pp. 589-604, 2006.

[22] D. M. Gaba, "The future vision of simulation in healthcare," Simulation in Healthcare, vol. 2, no. 2, pp. 126-135, 2007.

[23] D. D. Woods, "The alarm problem and directed attention in dynamic fault management," Ergonomics, vol. 38, no. 11, pp. 2371-2393, 1995. 